\title{
ISOMETRIC FACTORIZATION OF WEAKLY COMPACT OPERATORS AND THE APPROXIMATION PROPERTY
}

\author{
ÅSVALD LIMA, OLAV NYGAARD, AND EVE OJA
}

\begin{abstract}
Using an isometric version of the Davis, Figiel, Johnson, and Pełczyński factorization of weakly compact operators, we prove that a Banach space $X$ has the approximation property if and only if, for every Banach space $Y$, the finite rank operators of norm $\leq 1$ are dense in the unit ball of $\mathcal{W}(Y, X)$, the space of weakly compact operators from $Y$ to $X$, in the strong operator topology. We also show that, for every finite dimensional subspace $F$ of $\mathcal{W}(Y, X)$, there are a reflexive space $Z$, a norm one operator $J: Y \rightarrow Z$, and an isometry $\Phi: F \rightarrow \mathcal{W}(Z, X)$ which preserves finite rank and compact operators so that $T=\Phi(T) \circ J$ for all $T \in F$. This enables us to prove that $X$ has the approximation property if and only if the finite rank operators form an ideal in $\mathcal{W}(Y, X)$ for all Banach spaces $Y$.
\end{abstract}

\section{INTRODUCTION}

Let us recall that a linear subspace $F$ of a Banach space $E$ is an ideal in $E$ if $F^{\perp}$ is the kernel of a norm one projection in $E^{*}$. The notion of an ideal was introduced and studied by Godefroy, Kalton, and Saphar in [14].

J. Johnson [20] proved that if $X$ is a Banach space with the metric approximation property, then, for every Banach space $Y, \mathcal{F}(Y, X)$, the space of finite rank operators from $Y$ to $X$, is an ideal in $\mathcal{L}(Y, X)$, the space of bounded operators from $Y$ to $X$. Lima [23] has shown that the converse is true if $X$ has the Radon-Nikodým property. It is not known whether the converse is true in general.

Date: December 1998.

1991 Mathematics Subject Classification. Primary: 46B20, 46B28, $47 \mathrm{D} 15$.

Key words and phrases. Weakly compact operator, approximation property, DavisFigiel-Johnson-Pełczyński factorization.

The third-named author wishes to acknowledge the warm hospitality provided by Åsvald Lima and his colleagues at Agder College, where a part of this work was done in May-June 1998. Her visit was supported by the Norwegian Academy of Science and Letters and by Estonian Science Foundation Grant 3055. 
In [25], Lima and Oja proved that $X$ has the approximation property if and only if $\mathcal{F}(Y, X)$ is an ideal in $\mathcal{K}(Y, X)$, the space of compact operators from $Y$ to $X$, for all Banach spaces $Y$. In fact, they showed something stronger: $X$ has the approximation property if (and only if) $\mathcal{F}(Y, X)$ is an ideal in $\mathcal{K}(Y, X)$ for all separable reflexive spaces $Y$, or, equivalently, for all closed subspaces $Y$ of $c_{0}$.

It is natural to ask what happens if we look at $\mathcal{F}(Y, X)$ as a subspace of $\mathcal{W}(Y, X)$, the space of weakly compact operators from $Y$ to $X$, instead of looking at $\mathcal{F}(Y, X)$ as a subspace of $\mathcal{K}(Y, X)$. The answer to this question is the main result of this paper: $X$ has the approximation property if and only if $\mathcal{F}(Y, X)$ is an ideal in $\mathcal{W}(Y, X)$ for all Banach spaces $Y$, which in turn, is equivalent to the condition that, for every Banach space $Y$ and every $T \in \mathcal{W}(Y, X)$, there is a net $\left(T_{\alpha}\right)$ in $\mathcal{F}(Y, X)$ with $\sup _{\alpha}\left\|T_{\alpha}\right\| \leq\|T\|$ such that $T_{\alpha} y \rightarrow T y$ for all $y \in Y$.

We depart from the remarkable factorization theorem due to Davis, Figiel, Johnson, and Pełczyński [5] asserting that any weakly compact operator factors through a reflexive Banach space. In Section 1 (cf. Lemma 1.1), we make a quantitative change in the Davis-Figiel-JohnsonPełczyński construction which enables us to show, in Section 2, that one can factorize weakly compact operators through reflexive Banach spaces isometrically and even uniformly. In Theorem 1.2, we give a new characterization of the approximation property in terms of the Davis-FigielJohnson-Pełczyński factorization. We apply these results in Corollary 1.4 where we prove that $X$ has the approximation property if and only if every weakly compact operator into $X$ can be approximated in the strong operator topology by finite rank operators whose norms are at most equal to the norm of the weakly compact operator.

In Section 2 (cf. Lemma 2.1), we show that on the absolutely convex weakly compact set that is used in the factorization theorem of Davis, Figiel, Johnson, and Pełczyński to construct the reflexive Banach space, the two norm topologies coincide. (It was a part of the original construction that the two weak topologies coincide on the unit ball of the reflexive Banach space.) This, together with the quantitative modification of the Davis-Figiel-Johnson-Pełczyński construction made in Section 1, leads us to an isometric version of the Davis-Figiel-Johnson-Pełczyński factorization theorem (cf. Theorem 2.2). This also applies to show that the isometric factorization can even be uniform with respect to finite dimensional subspaces in the space of weakly compact operators (cf. Theorem 2.3 and Corollaries 2.4 and 2.5). 
We apply the uniform isometric factorization from Section 2 in Sections 3 and 4. Our main results in Section 3 are Theorem 3.3 and Theorem 3.4. They characterize the approximation property of $X$ and $X^{*}$ in terms of ideals of finite rank operators. In particular, Theorem 3.3 shows that $X$ has the approximation property if and only if $\mathcal{F}(Y, X)$ is an ideal in $\mathcal{W}(Y, X)$ for all Banach spaces $Y$, and Theorem 3.4 shows that $X^{*}$ has the approximation property if and only if $\mathcal{F}(X, Y)$ is an ideal in $\mathcal{W}(X, Y)$ for all Banach spaces $Y$.

In Section 4, an easy example shows that it is not possible to characterize the compact approximation property of $X$ by $\mathcal{K}(Y, X)$ being an ideal in $\mathcal{W}(Y, X)$ for all $Y$ (although this property characterizes the compact approximation property for reflexive $X)$. In Theorem 4.1, we give some conditions equivalent to $\mathcal{K}(Y, X)$ being an ideal in $\mathcal{W}(Y, X)$ for all $Y$. We also show, by using the description of duals of spaces of compact operators due to Feder and Saphar [12], that these conditions are implied by the compact approximation property of $X$ (cf. also Theorem 4.1).

In Theorems 5.1 and 5.2 of the final Section 5, we demonstrate how the method of proof of Theorem 1.2 can be further developed to give alternative proofs (through ideals of finite rank or compact operators) for known results about cases when the (compact) approximation property implies the metric (compact) approximation property. In particular, as an immediate corollary, we obtain the result due to Godefroy and Saphar [15] that $X^{*}$ has the metric compact approximation property with conjugate operators whenever $X^{*}$ has the compact approximation property with conjugate operators and $X^{*}$ or $X^{* *}$ has the Radon-Nikodým property.

Let us fix some more notation. In a linear normed space $X$, we denote the closed unit ball by $B_{X}$ and the closed ball with center $x$ and radius $r$ by $B_{X}(x, r)$. For a set $A \subset X$, its norm closure is denoted by $\bar{A}$, its linear span by span $A$, its convex hull by conv $A$, and the set of its strongly exposed points by $\operatorname{sexp} A$.

We shall write $\mathcal{K}_{X}$ (resp. $\mathcal{W}_{X}$ ) for the family of all compact (resp. weakly compact) absolutely convex subsets of $B_{X}$.

\section{Criteria of the approximation property in terms of the DAVIS-FIGIEL-JOHNSON-PEECZYŃSKI FACTORIZATION}

In this section, we depart from the famous Davis, Figiel, Johnson, and Pełczyński factorization construction (cf. Lemma 1 on p. 313 in [5], [6, pp. 160-161], [7, p. 227], [33, p. 51] or Lemma 1.1 below) and apply the Grothendieck-Feder-Saphar description of duals of spaces of compact 
operators (cf. [16] or [8] and [12]) to obtain several conditions equivalent to the approximation property of Banach spaces, all of them expressed in terms of the Davis-Figiel-Johnson-Pełczyński construction (cf. Theorem 1.2 below). This leads us to an interesting "metric" characterization of the approximation property (cf. Corollary 1.4) similar to the well-known characterization of the metric approximation property as the denseness of $B_{\mathcal{F}(Y, X)}$ in $B_{\mathcal{L}(Y, X)}$ in the topology of uniform convergence on compact sets, for all Banach spaces $Y$.

We shall need a quantitative version of the classical Davis, Figiel, Johnson, Pełczyński factorization construction, which in fact consists in replacing the number 2 in the original construction by $\sqrt{a}$ for any $a>1$. We now fix the notation to describe the Davis-Figiel-Johnson-Pełczyński construction, and we shall also use this notation in the following sections.

Let $a>1$. Let $X$ be a Banach space and let $K$ be a closed absolutely convex subset of its unit ball $B_{X}$. For each $n \in \mathbb{N}=\{1,2, \ldots\}$, put $B_{n}=a^{n / 2} K+a^{-n / 2} B_{X}$ and denote by \|\|$_{n}$ the equivalent norm on $X$ defined by the gauge of $B_{n}$. Let $\|x\|_{K}=\left(\sum_{n=1}^{\infty}\|x\|_{n}^{2}\right)^{1 / 2}, X_{K}=\{x \in$ $\left.X:\|x\|_{K}<\infty\right\}$ and $C_{K}=\left\{x \in X:\|x\|_{K} \leq 1\right\}$. Further, let $J_{K}$ denote the identity embedding of $X_{K}$ into $X$. Finally, we put

$$
f(a)=\left(\sum_{n=1}^{\infty} \frac{a^{n}}{\left(a^{n}+1\right)^{2}}\right)^{1 / 2}
$$

and note that $f:(1, \infty) \rightarrow \mathbb{R}$ is a continuous, strictly decreasing function with $\lim _{a \rightarrow 1^{+}} f(a)=\infty$ and $\lim _{a \rightarrow \infty} f(a)=0$. Hence, there is a unique point $\tilde{a} \in(1, \infty)$ such that $f(\tilde{a})=1$. (A "good" estimate of this $\tilde{a}$ is $\exp (4 / 9)=1.55962349761 \ldots)$ For this $\tilde{a}$, one has $K \subset C_{K} \subset B_{X}$ (this is clear from Lemma 1.1 below).

The following is the classical Davis-Figiel-Johnson-Pełczyński factorization lemma with some "cosmetic" changes.

Lemma 1.1 (cf. p. 313 in [5]).

(i) $K \subset f(a) C_{K}$.

(ii) $X_{K}$ is a Banach space with the closed unit ball $C_{K}$, and $J_{K} \in$ $\mathcal{L}\left(X_{K}, X\right)$, and $\left\|J_{K}\right\| \leq 1 / f(a)$.

(iii) $J_{K}^{* *}$ is injective.

(iv) $X_{K}$ is reflexive if and only if $K$ is weakly compact.

Proof. Only (i) and $\left\|J_{K}\right\| \leq 1 / f(a)$ in (ii) need to be verified.

Suppose $x \in K$. Since $x \in B_{X}$, we get

$$
a^{n / 2} x+a^{-n / 2} x \in B_{n},
$$


so that

$$
\|x\|_{n} \leq \frac{1}{a^{n / 2}+a^{-n / 2}}=\frac{a^{n / 2}}{a^{n}+1}
$$

for all $n$. Hence $\|x\|_{K} \leq f(a)$. This proves (i).

Since $B_{X}$ is convex and $K \subset B_{X}$, we have

$$
\frac{1}{a^{n / 2}+a^{-n / 2}}\left(a^{n / 2} K+a^{-n / 2} B_{X}\right) \subset B_{X},
$$

that is

$$
\frac{a^{n / 2}}{a^{n}+1} B_{n} \subset B_{X}
$$

Hence

$$
\|x\|_{n} \geq \frac{a^{n / 2}}{a^{n}+1}\|x\|
$$

and therefore $\|x\|_{K} \geq f(a)\|x\|$ for all $x \in X_{K}$, meaning that $\left\|J_{K}\right\| \leq$ $1 / f(a)$.

Theorem 1.2. For a Banach space $X$, the following assertions are equivalent.

(i) $X$ has the approximation property.

(ii) $\mathcal{F}\left(X_{K}, X\right)$ is an ideal in $\mathcal{L}\left(X_{K}, X\right)$ for every $K \in \mathcal{W}_{X}$.

(iii) For every $K \in \mathcal{W}_{X}$, there exists a net $\left(A_{\alpha}\right)$ in $\mathcal{F}\left(X_{K}, X\right)$ with $\sup _{\alpha}\left\|A_{\alpha}\right\| \leq\left\|J_{K}\right\|$ such that $A_{\alpha} x \underset{\alpha}{\longrightarrow} J_{K} x$ for all $x \in X_{K}$.

(iv) For every $K \in \mathcal{W}_{X}$, there exists a bounded net $\left(A_{\alpha}\right)$ in $\mathcal{F}\left(X_{K}, X\right)$ such that $A_{\alpha} x \underset{\alpha}{\longrightarrow} J_{K} x$ for all $x \in X_{K}$.

(v) For every $K \in \mathcal{K}_{X}$, there exists a net $\left(A_{\alpha}\right)$ in $\mathcal{F}\left(X_{K}, X\right)$ such that $\left\|A_{\alpha}-J_{K}\right\| \underset{\alpha}{\longrightarrow} 0$.

Remark 1.1. Condition (v) means that $J_{K}$ belongs to the norm closure of $\mathcal{F}\left(X_{K}, X\right)$ in $\mathcal{L}\left(X_{K}, X\right)$ and (iii) can be viewed as its "metric" version: $J_{K}$ belongs to the closure of the ball $\mathcal{F}\left(X_{K}, X\right) \cap B\left(0,\left\|J_{K}\right\|\right)$ in the strong operator topology of $\mathcal{L}\left(X_{K}, X\right)$.

The proof of Theorem 1.2, as well as some other proofs of this paper, will use the following result.

Lemma 1.3. Let $X$ and $Y$ be Banach spaces. Let $\mathcal{A}$ be a subspace of $\mathcal{L}(Y, X)$ containing $\mathcal{F}(Y, X)$ and let $T \in \mathcal{L}(Y, X)$. If $\mathcal{A}$ is an ideal in 
$\mathcal{L}:=\operatorname{span}(\mathcal{A} \cup\{T\})$ and $P$ is an ideal projection, then there exists a net $\left(A_{\alpha}\right) \subset \mathcal{A}$ with $\sup _{\alpha}\left\|A_{\alpha}\right\| \leq\|T\|$ such that

$$
y^{* *}\left(A_{\alpha}^{*} x^{*}\right) \underset{\alpha}{\rightarrow}\left(P\left(y^{* *} \otimes x^{*}\right)\right)(T) \quad \text { for all } x^{*} \in X^{*} \text { and } y^{* *} \in Y^{* *} .
$$

Moreover, if $Y$ has the Radon-Nikodym property (in particular, if $Y$ is reflexive), then $\left(A_{\alpha}\right)$ can be chosen to satisfy

$$
A_{\alpha} y \rightarrow \text { Ty for all } y \in Y \text {. }
$$

Proof. Let $P$ be a norm one projection on $\mathcal{L}^{*}$ with $\operatorname{ker} P=\mathcal{A}^{\perp}$. Since $P^{*}(T) \in \mathcal{A}^{\perp \perp} \subset \mathcal{L}^{* *}$ and $\left\|P^{*}(T)\right\| \leq\|T\|$, there exists a net $\left(A_{\alpha}\right) \subset \mathcal{A}$ with $\sup _{\alpha}\left\|A_{\alpha}\right\| \leq\|T\|$ such that $A_{\alpha} \rightarrow P^{*}(T)$ weak* in $\mathcal{L}^{* *}$. In particular, for $x^{*} \in X^{*}$ and $y^{* *} \in Y^{* *}$, we have

$$
y^{* *}\left(A_{\alpha}^{*} x^{*}\right)=\left(y^{* *} \otimes x^{*}\right)\left(A_{\alpha}\right) \underset{\alpha}{\longrightarrow}\left(y^{* *} \otimes x^{*}\right)\left(P^{*}(T)\right)=\left(P\left(y^{* *} \otimes x^{*}\right)\right)(T) .
$$

It is straightforward to verify that, for any $f \in \mathcal{L}^{*}, P f$ is a normpreserving extension of $\left.f\right|_{\mathcal{A}} \in \mathcal{A}^{*}$. On the other hand, it is proved in [24, Lemma 3.4, (b)] that $y \otimes x^{*} \in \mathcal{F}(Y, X)^{*}$ has a unique norm-preserving extension to the whole $\mathcal{L}(Y, X)$ whenever $x^{*} \in X^{*}$ and $y \in \operatorname{sexp} B_{Y}$. Therefore $P\left(y \otimes x^{*}\right)=y \otimes x^{*} \in \mathcal{L}^{*}$ and

$$
\left(A_{\alpha}^{*} x^{*}\right)(y) \underset{\alpha}{\rightarrow}\left(y \otimes x^{*}\right)(T)=\left(T^{*} x^{*}\right)(y) \text { for all } x^{*} \in X^{*} \text { and } y \in \operatorname{sexp} B_{Y} \text {. }
$$

If $Y$ has the Radon-Nikodým property, then $Y=\overline{\operatorname{span}}\left(\operatorname{sexp} B_{Y}\right)$, and we get that

$$
\left(A_{\alpha}^{*} x^{*}\right)(y) \underset{\alpha}{\longrightarrow}\left(T^{*} x^{*}\right)(y) \text { for all } x^{*} \in X^{*} \text { and } y \in Y \text {. }
$$

This means that $A_{\alpha} \rightarrow T$ in the weak operator topology of $\mathcal{L}(Y, X)$. Since the weak and strong operator topologies yield the same dual space (cf. e.g. [9, Theorem VI.1.4]), after passing to convex combinations, we may assume that $A_{\alpha} \rightarrow T$ strongly.

Proof of Theorem 1.2. (i) $\Rightarrow$ (ii). Since $X_{K}$ is reflexive (cf. Lemma 1.1) and $X$ has the approximation property, by a classical representation theorems due to Grothendieck [16] (cf. also e.g. [8, Chapter VIII]), we have $\mathcal{F}\left(X_{K}, X\right)^{*}=X^{*} \hat{\otimes}_{\pi} X_{K}$, the duality between $\mathcal{F}\left(X_{K}, X\right)$ and $X^{*} \hat{\otimes}_{\pi} X_{K}$ being expressed by the formula

$$
v(T)=\operatorname{trace}(T v), \quad T \in \mathcal{F}\left(X_{K}, X\right), \quad v \in X^{*} \hat{\otimes}_{\pi} X_{K} .
$$

We also have a natural linear transformation $\Phi: X^{*} \hat{\otimes}_{\pi} X_{K} \rightarrow \mathcal{L}\left(X_{K}, X\right)^{*}$ defined by

$$
(\Phi v)(T)=\operatorname{trace}(T v), \quad T \in \mathcal{L}\left(X_{K}, X\right), \quad v \in X^{*} \hat{\otimes}_{\pi} X_{K} .
$$


It is clear that $\|\Phi\| \leq 1$ (because $|\operatorname{trace}(T v)| \leq\|T v\|_{\pi} \leq\|T\|\|v\|_{\pi}$ ). Since $\Phi v$ extends $v$, we have $\|v\| \leq\|\Phi v\| \leq\|v\|$, hence $\|\Phi v\|=\|v\|$ for all $v \in \mathcal{F}\left(X_{K}, X\right)^{*}=X^{*} \hat{\otimes}_{\pi} X_{K}$. It is straightforward to verify that $P: \mathcal{L}\left(X_{K}, X\right)^{*} \rightarrow \mathcal{L}\left(X_{K}, X\right)^{*}$ defined by

$$
P f=\Phi\left(\left.f\right|_{\mathcal{F}\left(X_{K}, X\right)}\right), \quad f \in \mathcal{L}\left(X_{K}, X\right)^{*},
$$

is the desired ideal projection.

(ii) $\Rightarrow$ (iii). This is immediate from Lemma 1.3 because $X_{K}$ is reflexive.

(iii) $\Rightarrow$ (iv). This is obvious.

(iv) $\Rightarrow(\mathrm{v})$. Let $K \in \mathcal{K}_{X}$. Then $J_{K} \in \mathcal{K}\left(X_{K}, X\right)$ because

$$
J_{K}\left(C_{K}\right)=C_{K} \subset a^{n / 2} K+a^{-n / 2} B_{X} \text {, for all } n \in \mathbb{N} \text {, }
$$

implies that $J_{K}\left(C_{K}\right)$ has, for any $\varepsilon>0$, a finite $\varepsilon$-net and therefore it is relatively compact in $X$. By the description of the weak convergence in spaces of compact operators due to Feder and Saphar [12, Corollary 1.2] (the reflexivity of $X_{K}$ and the boundedness of $\left(A_{\alpha}\right)$ are used here), we get that $\left(A_{\alpha}-J_{K}\right) \rightarrow 0$ weakly in $\mathcal{K}\left(X_{K}, X\right)$. After passing to convex combinations, we may assume that $\left\|A_{\alpha}-J_{K}\right\| \rightarrow 0$.

$(\mathrm{v}) \Rightarrow(\mathrm{i})$. Let $K$ be a compact subset of $X$ and let $\varepsilon>0$. We have to show that there is an operator $T \in \mathcal{F}(X, X)$ such that $\|T x-x\|<\varepsilon$ for all $x \in K$. We may assume that $K \in \mathcal{K}_{X}$ (note that, by a theorem of Mazur, the absolutely convex hull of a compact set in a Banach space is compact). By (v), there is an operator $A=\sum_{i=1}^{n} y_{i}^{*} \otimes x_{i} \in \mathcal{F}\left(X_{K}, X\right)$ (with $y_{i}^{*} \in$ $\left.X_{K}^{*}, x_{i} \in X\right)$ such that $\left\|A-J_{K}\right\|<\varepsilon / 2 f(a)$. Since $J_{K}^{* *}$ is injective (cf. Lemma 1.1), $J_{K}^{*}\left(X^{*}\right)$ is norm dense in $X_{K}^{*}$. Let $x_{i}^{*} \in X^{*}$ satisfy $\left\|y_{i}^{*}-J_{K}^{*} x_{i}^{*}\right\|<\varepsilon / 2 f(a) \sum_{i=1}^{n}\left\|x_{i}\right\|$ and let $T=\sum_{i=1}^{n} x_{i}^{*} \otimes x_{i} \in \mathcal{F}(X, X)$. Then, for every $x \in K$ (recall from Lemma 1.1 that $K \subset f(a) C_{K}$ ), we have

$$
\begin{aligned}
\|T x-x\| & =\left\|T J_{K} x-J_{K} x\right\| \\
& \leq\left\|A-J_{K}\right\|\|x\|_{K}+\left\|T J_{K}-A\right\|\|x\|_{K} \\
& <\frac{\varepsilon}{2}+f(a)\left\|\sum_{i=1}^{n}\left(J_{K}^{*} x_{i}^{*}-y_{i}^{*}\right) \otimes x_{i}\right\| \\
& \leq \frac{\varepsilon}{2}+f(a) \sum_{i=1}^{n}\left\|J_{K}^{*} x_{i}^{*}-y_{i}^{*}\right\|\left\|x_{i}\right\|<\frac{\varepsilon}{2}+\frac{\varepsilon}{2}=\varepsilon
\end{aligned}
$$

Remark 1.2. A famous theorem due to Grothendieck [16] (cf. e.g. [26, p. 32]) asserts that $X$ has the approximation property if and only if $\overline{\mathcal{F}(Y, X)}=\mathcal{K}(Y, X)$ for all Banach spaces $Y$. Here the "only if" part is 
easy and straightforward (cf. e.g. [26, p. 32]). The "classical" proof of the "if" part relies on Grothendieck's characterization of a compact set as a subset of the closed convex hull of a norm-null sequence (cf. e.g. [26, pp. 32-33]) which is used to construct a Banach space $Y$ - a linear subspace of $X$ - such that the formal identity map from $Y$ into $X$ is compact. The proof of the implication (v) $\Rightarrow$ (i) above provides an alternative easier proof to the "if" part (where $X_{K}$ together with the identity map $J_{K}$ plays the role of $Y$ ). And combined together with Theorem (AP) in [25], this also gives an easy short proof for the classical fact (due to Grothendieck) that $X^{*}$ has the approximation property if and only if $\overline{\mathcal{F}(X, Y)}=\mathcal{K}(X, Y)$ for all Banach spaces $Y$.

A Banach space $X$ has the approximation property if and only if, for every Banach space $Y$, the finite rank operators are dense in $\mathcal{L}(Y, X)$ in the topology $\tau$ of uniform convergence on compact sets, and $X$ has the metric approximation property if and only if the "metric" version of this condition holds: for every Banach space $Y$, the finite rank operators of norm $\leq 1$ are dense in the unit ball of $\mathcal{L}(Y, X)$ in the topology $\tau$ (cf. e.g. $[26$, pp. 32,39$])$. The next result provides a similar "metric" criterion for the approximation property.

Corollary 1.4. For a Banach space $X$, the following assertions are equivalent.

(i) $X$ has the approximation property.

(ii) For every Banach space $Y, B_{\mathcal{F}(Y, X)}$ is dense in $B_{\mathcal{W}(Y, X)}$ in the strong operator topology.

(iii) For every Banach space $Y$ and every $T \in \mathcal{W}(Y, X)$, there is a net $\left(T_{\alpha}\right)$ in $\mathcal{F}(Y, X)$ with $\sup _{\alpha}\left\|T_{\alpha}\right\| \leq\|T\|$ such that $T_{\alpha} y \rightarrow T y$ for all $y \in Y$.

(iv) For every separable reflexive Banach space $Y$ and every $T \in$ $\mathcal{W}(Y, X)$ there is a sequence $\left(T_{n}\right)$ in $\mathcal{F}(Y, X)$ with $\sup _{n}\left\|T_{n}\right\| \leq$ $\|T\|$ such that $T_{n} y \rightarrow T y$ for all $y \in Y$.

Proof. (i) $\Rightarrow$ (iii). We may assume that $\|T\|=1$. Then $K:=\overline{T\left(B_{Y}\right)} \in$ $\mathcal{W}_{X}$. Let the number $a$ be fixed so that $f(a)=1$. Then (cf. Lemma 1.1) $T\left(B_{Y}\right) \subset C_{K}=B_{X_{K}}$ and $\left\|J_{K}\right\| \leq 1$. By Theorem $1.2((\mathrm{i}) \Rightarrow($ iii $))$, there exists a net $\left(A_{\alpha}\right)$ in $\mathcal{F}\left(X_{K}, X\right)$ with $\sup _{\alpha}\left\|A_{\alpha}\right\| \leq 1$ such that $A_{\alpha} x \rightarrow J_{K} x$ for all $x \in X_{K}$. Define $T_{\alpha}: Y \rightarrow X$ by $T_{\alpha} y=A_{\alpha} T y, y \in Y$. Then $T_{\alpha}: Y \rightarrow X$ is linear and of finite rank, $T_{\alpha} y \rightarrow T y$ for all $y \in Y$, and $\left\|T_{\alpha}\right\| \leq \sup \left\{\|T y\|_{K}: y \in B_{Y}\right\} \leq 1$ for all $\alpha$.

(iii) $\Rightarrow$ (ii). This is obvious. 
(ii) $\Rightarrow$ (i). By Lemma 1.1, $J_{K} \in \mathcal{W}\left(X_{K}, X\right)$ whenever $K \in \mathcal{W}_{X}$ (because $X_{K}$ is reflexive). Therefore, (ii) implies assertion (iv) of Theorem 1.2, which is equivalent to (i).

(iii) $\Rightarrow$ (iv). Let $Y$ be a separable Banach space and let $T \in \mathcal{W}(Y, X)$. Let $\left(y_{n}\right)$ be a dense sequence in $B_{Y}$. By a standard argument, picking from the given net $\left(T_{\alpha}\right)$, for each $n=1,2, \ldots$, operators $T_{\alpha_{n}}$ so that $\left\|T_{\alpha_{n}} y_{1}-T y_{1}\right\|<1 / n, \ldots,\left\|T_{\alpha_{n}} y_{n}-T y_{n}\right\|<1 / n$, one obtains the desired sequence $\left(T_{n}\right)=\left(T_{\alpha_{n}}\right)$.

(iv) $\Rightarrow$ (i). Let $Z$ be any reflexive Banach space and let $T \in \mathcal{W}(Z, X)$. Recall that every separable subspace of $Z$ is contained in a separable 1-complemented subspace $Y$ of $Z$, meaning that there exists a norm one projection $P_{Y}$ from $Z$ onto $Y$ (this so-called "separable 1-complementation property" is shared by all weakly compactly generated spaces (cf. [1] or e.g. $[6$, p. 149])). Therefore the set of all triples $\alpha=(F, Y, \varepsilon)$, where $F$ is a finite dimensional subspace of $Z, Y$ is a separable 1-complemented subspace of $Z$ containing $F$, and $\epsilon>0$, is a directed set in the natural way. For any $\alpha=(F, Y, \varepsilon)$, considering $\left.T\right|_{Y} \in \mathcal{W}(Y, X)$, we choose an operator $T_{\varepsilon} \in \mathcal{F}(Y, X)$ with $\left\|T_{\varepsilon}\right\| \leq\left\|\left.T\right|_{Y}\right\|$ such that $\left\|T_{\varepsilon} y-T y\right\|<\varepsilon$ for all $y \in B_{F}$ and let $T_{\alpha}=T_{\varepsilon} \circ P_{Y}$. Then $\left(T_{\alpha}\right) \subset \mathcal{F}(Z, X)$ satisfies $\sup _{\alpha}\left\|T_{\alpha}\right\| \leq\|T\|$ and $T_{\alpha} z \rightarrow T z$ for all $z \in Z$. In particular, this gives assertion (iii) of Theorem 1.2 which is equivalent to (i).

Remark 1.3. Concerning the implication (i) $\Rightarrow$ (ii) of Corollary 1.4, we note that, by a result due to Grothendieck [16, Corollary 2, p. 141], the approximation property of the dual space $X^{*}$ implies condition (ii) of Corollary 1.4. We are grateful to the Referee for pointing out this for us. Grothendieck's proof relies on his theorem stating that if $A$ and $B$ are, respectively, integral and weakly compact operators, then $A B$ is a nuclear operator with the nuclear norm not greater than $\|B\|$ multiplied by the integral norm of $A$.

\section{UNIFORM ISOMETRIC FACTORIZATION}

The remarkable factorization theorem due to Davies, Figiel, Johnson, and Pełczyński [5] asserts that any weakly compact operator $T$ factors through a reflexive space. In this case, if we write $T=A \circ B$, it is clear that the operators $A$ and $B$ are weakly compact. By a theorem of Figiel and Johnson ([13] and [21]), if $T$ is a compact operator, then it admits a factorization $T=A \circ B$ where $A$ and $B$ are compact. (This fact can also be deduced from the Davis-Figiel-Johnson-Pełczyński theorem (cf. e.g. $[19$, p. 374]).) 
In Theorem 2.2 below, we shall see that the quantitative modification in the Davis-Figiel-Johnson-Pełczyński construction made in Section 1, together with the following Lemma 2.1, leads to an isometric factorization in the Davis-Figiel-Johnson-Pełczyński and the Figiel-Johnson theorems. (In particular, if $\|T\|=1$, then $\|A\|=\|B\|=1$; the estimates from [33, p. 51] would give $\|A\|,\|B\| \leq 4$.)

Lemma 2.1 (Lemma 1.1 continued).

(i) For $x \in K$, one has

$$
\|x\|_{K}^{2} \leq\left(\frac{1}{4}+\frac{1}{\ln a}\right)\|x\| .
$$

(ii) The $X$-norm and $X_{K}$-norm topologies coincide on $K$.

(iii) The weak topologies defined by $X^{*}$ and $X_{K}^{*}$ coincide on $C_{K}$.

(iv) $C_{K}$ as a subset of $X$ is compact, weakly compact, or separable if and only if $K$ has the same property.

Proof. (i) Let $x \in K, x \neq 0$. Then we have

$$
a^{n / 2} x+a^{-n / 2} \frac{x}{\|x\|} \in B_{n},
$$

so that

$$
\|x\|_{K}^{2} \leq \sum_{n=1}^{\infty} \frac{1}{\left(a^{n / 2}+a^{-n / 2}\|x\|^{-1}\right)^{2}}=\|x\| \sum_{n=1}^{\infty} \frac{a^{n}\|x\|}{\left(a^{n}\|x\|+1\right)^{2}} .
$$

Let $h(t)=a^{t}\|x\| /\left(a^{t}\|x\|+1\right)^{2}, 1 \leq t<\infty$. The graph of $h$ has a bellshaped form and $\max h(t)=1 / 4$. Let $k \in \mathbb{N}$ be such that

$$
h(1) \leq h(2) \leq \cdots \leq h(k-1) \leq h(k) \geq h(k+1) \geq \cdots .
$$

Then

$$
\begin{aligned}
\frac{\|x\|_{K}^{2}}{\|x\|} & \leq \sum_{n=1}^{\infty} h(n) \leq h(k)+\int_{1}^{\infty} h(t) d t \\
& \leq \frac{1}{4}+\frac{1}{\ln a} \int_{1+a\|x\|}^{\infty} \frac{d u}{u^{2}} \\
& =\frac{1}{4}+\frac{1}{\ln a}\left(\frac{1}{1+a\|x\|}\right) \leq \frac{1}{4}+\frac{1}{\ln a} .
\end{aligned}
$$

(ii) For $x, y \in K$, we have $\frac{x-y}{2} \in K$. By (i),

$$
\|x-y\|_{K}^{2} \leq\left(\frac{1}{2}+\frac{2}{\ln a}\right)\|x-y\| .
$$

This together with (ii) in Lemma 1.1 gives (ii). 
(iii) This is proved in [5].

(iv) This is essentially known (cf. [5] or [7, p. 228]) and follows from the inclusions $(f(a))^{-1} K \subset C_{K} \subset a^{n / 2} K+a^{-n / 2} B_{X}$, for all $n$, and from the fact that $C_{K}=\bigcap_{n=1}^{\infty}\left\{x \in X: \sum_{k=1}^{n}\|x\|_{k}^{2} \leq 1\right\}$ is closed and weakly closed.

Theorem 2.2. Suppose $T \in \mathcal{L}(Y, X)$. Let $K=\overline{T\left(B_{Y}(0,1 /\|T\|)\right)}$ and let $T_{K}: Y \rightarrow X_{K}$ be defined by $T_{K} y=T y, y \in Y$. Then $T=J_{K} \circ T_{K}$ and

(i) $T$ is separably valued, weakly compact, compact, or of finite rank if and only if $T_{K}$ has the same property if and only if $J_{K}$ has the same property.

(ii) $\|T\|=\left\|T_{K}\right\|$ and $\left\|J_{K}\right\|=1$ whenever $f(a)=1$.

Proof. (i) We only need to prove that the above-mentioned properties of $T$ imply the same properties for $T_{K}$ and $J_{K}$. Since $T_{K}$ is algebraically the same operator as $T$, they have the same rank and, by Lemma 2.1, (ii) and (iii), $T_{K}$ is separably valued, compact, or weakly compact whenever $T$ is. If $T$ is of finite rank, then $J_{K}$ has finite rank since

$$
J_{K}\left(B_{X_{K}}\right)=C_{K} \subset \bigcap_{n=1}^{\infty}\left(T(Y)+a^{-n / 2} B_{X}\right)=\overline{T(Y)}=T(Y) .
$$

That the other properties of $T$ imply the same properties for $J_{K}$, it is clear from Lemma 2.1, (iv).

(ii) If $f(a)=1$, then $\left\|J_{K}\right\| \leq 1$ by Lemma 1.1, (ii). Without loss of generality, we may assume that $\|T\|=1$. Since $K \subset C_{K}$ (cf. Lemma 1.1), (i), we get $\left\|T_{K}\right\|=\sup _{y \in B_{Y}}\|T y\|_{K} \leq \sup _{z \in K}\|z\|_{K} \leq \sup _{z \in C_{K}}\|z\|_{K}=1$. But then

$$
1=\|T\|=\left\|J_{K} \circ T_{K}\right\| \leq\left\|J_{K}\right\|\left\|T_{K}\right\| \leq \min \left\{\left\|T_{K}\right\|,\left\|J_{K}\right\|\right\} .
$$

Therefore $\left\|T_{K}\right\|=\left\|J_{K}\right\|=1$.

By developing the method of proof of Theorem 2.2, we shall show (cf. Theorem 2.3 and Corollaries 2.4 and 2.5) that the isometric factorization can even be uniform with respect to finite dimensional subspaces in the space of weakly compact operators.

Theorem 2.3. Let $F$ be a finite dimensional subspace of $\mathcal{W}(Y, X)$. Then there exist a reflexive space $Z$, a norm one operator $J: Z \rightarrow X$, and a linear isometry $\Phi: F \rightarrow \mathcal{W}(Y, Z)$ such that $T=J \circ \Phi(T)$ for all $T \in F$. Moreover,

(i) $Z=X_{K}$ and $J=J_{K}$ for some $K \in \mathcal{W}_{X}$ whenever the number a is fixed so that $f(a)=1$, 
(ii) $T$ is compact if and only if $\Phi(T)$ is compact,

(iii) $T$ has finite rank if and only if $\Phi(T)$ has finite rank.

Proof. Let $K=\overline{\operatorname{conv}}\left\{T y: T \in B_{F}\right.$ and $\left.y \in B_{Y}\right\}$. Then $K$ is a weakly closed absolutely convex subset of $B_{X}$. We shall use Grothendieck's lemma (cf. e.g. [7, p. 227]) to show that $K$ is weakly compact. For given $\varepsilon>0$, let $\left\{T_{1}, \ldots, T_{n}\right\}$ be an $\varepsilon / 2$-net of $B_{F}$. Let $K_{\varepsilon}$ be the closed convex hull of the weakly compact set $\overline{T_{1}\left(B_{Y}\right)} \cup \ldots \cup \overline{T_{n}\left(B_{Y}\right)}$. By the Krein-Šmulian theorem, $K_{\varepsilon}$ is weakly compact. Since $K \subset K_{\varepsilon}+\varepsilon B_{X}$, the weak compactness of $K$ follows from Grothendieck's lemma.

Choose $a$ such that $f(a)=1$. Put $Z=X_{K}, J=J_{K}$, and define $\Phi: F \rightarrow \mathcal{W}(Y, Z)$ by $\Phi(T) y=T y, y \in Y$. Then $Z$ is reflexive (since $K$ is weakly compact), $\Phi$ is linear, and $T=J \circ \Phi(T)$ for all $T \in F$. As in the proof of Theorem 2.2, we show (i) and (ii), and we also obtain that $\|\Phi(T)\|=1$, whenever $\|T\|=1$, and that $\|J\|=1$.

Remark 2.1. The proof of Theorem 2.3 shows how norm compact sets in the space of weakly compact operators can be uniformly and isometrically factorized.

Corollary 2.4. Let $F$ be a finite dimensional subspace of $\mathcal{W}(X, Y)$. Then there exist a reflexive space $Z$, a norm one operator $J: X \rightarrow Z$, and a linear isometry $\Phi: F \rightarrow \mathcal{W}(Z, Y)$ such that $T=\Phi(T) \circ J$ for all $T \in F$. Moreover,

(i) $T$ is compact if and only if $\Phi(T)$ is compact,

(ii) $T$ has finite rank if and only if $\Phi(T)$ has finite rank.

Proof. Let us consider the finite dimensional subspace $G=\left\{T^{*}: T \in F\right\}$ of $\mathcal{W}\left(Y^{*}, X^{*}\right)$. By Theorem 2.3, there exist a reflexive space $Z$, a norm one operator $I: Z^{*} \rightarrow X^{*}$, and a linear isometry $\Psi: G \rightarrow \mathcal{W}\left(Y^{*}, Z^{*}\right)$ so that $T^{*}=I \circ \Psi\left(T^{*}\right)$ for all $T \in F$. Put $J=\left.I^{*}\right|_{X}$ and define $\Phi(T)=\left(\Psi\left(T^{*}\right)\right)^{*}$ for $T \in F$. Since $\left.T^{* *}\right|_{X}=T$ whenever $T \in F$, we have $T=\Phi(T) \circ J$ and $\Phi(T) \in \mathcal{W}(Z, Y)$ for all $T \in F$. Moreover, $\|\Phi(T)\|=\left\|\left(\Psi\left(T^{*}\right)\right)^{*}\right\|=$ $\left\|\Psi\left(T^{*}\right)\right\|=\left\|T^{*}\right\|=\|T\|$ for $T \in F$. The linearity of $\Phi$ and properties (i) and (ii) are also clear from the definition of $\Phi$. Finally, it is easily seen that $\|J\|=1$.

Corollary 2.4 will be applied in the next section to prove that $\mathcal{F}(Y, X)$ is an ideal in $\mathcal{W}(Y, X)$ for all Banach spaces $Y$ whenever $X$ has the approximation property. We conclude this section with an immediate corollary from Theorem 2.3 and Corollary 2.4.

Corollary 2.5. For every finite dimensional subspace $F$ of $\mathcal{W}(X, Y)$, there exist reflexive spaces $Z$ and $W$, norm one operators $J: X \rightarrow Z$ 
and $I: W \rightarrow Y$, and a linear isometry $\Phi: F \rightarrow \mathcal{W}(Z, W)$ such that $T=I \circ \Phi(T) \circ J$ for all $T \in F$.

\section{The APPROXIMATION PROPERTY AND IDEALS OF FINITE RANK OPERATORS}

In this section, our main objective is to prove that a Banach space $X$ has the approximation property if and only if $\mathcal{F}(Y, X)$ is an ideal in $\mathcal{W}(Y, X)$ for all Banach spaces $Y$ (see Theorem 3.3 below which also lists other criteria of the approximation property in terms of ideals of finite rank operators). In fact, we have already proved (see Theorem 1.2 and the proof of its implication (i) $\Rightarrow$ (ii)) that $X$ has the approximation property if and only if $\mathcal{F}(Y, X)$ is an ideal in $\mathcal{W}(Y, X)$ for all reflexive Banach spaces $Y$. The next result extends this assertion from reflexive spaces to all Banach spaces.

Theorem 3.1. Let $X$ be a Banach space. Then $\mathcal{F}(Y, X)$ (resp. $\mathcal{K}(Y, X))$ is an ideal in $\mathcal{W}(Y, X)$ for all Banach spaces $Y$ if and only if $\mathcal{F}(Z, X)$ (resp. $\mathcal{K}(Z, X))$ is an ideal in $\mathcal{W}(Z, X)$ for all reflexive spaces $Z$.

The proof of Theorem 3.1 will use the uniform isometric factorization of weakly compact operators from Section 2 and the following alternative characterization of ideals (proved e.g. in Lima [23], Fakhoury [11], and Kalton [22]).

Theorem 3.2. Let $F$ be a closed subspace of a Banach space E. The following statements are equivalent.

(i) $F$ is an ideal in $E$.

(ii) $F$ is locally 1-complemented in $E$, i.e. for every finite dimensional subspace $G$ of $E$ and for all $\varepsilon>0$, there is an operator $A: G \rightarrow F$ such that $\|A\|<1+\varepsilon$ and $A x=x$ for all $x \in G \cap F$.

Remark 3.1. It is straightforward to verify that the condition $A x=x$ for all $x \in G \cap F$ in Theorem 3.2 can be replaced by $\|A x-x\| \leq \varepsilon$ for all $x \in B_{G \cap F}$.

Let us recall that, for a linear subspace $F$ of a Banach space $E$ (as it is clear from the definition of the ideal), $F$ is an ideal in $E$ if and only if $\bar{F}$ is an ideal in $E$.

Proof of Theorem 3.1. We shall first consider the case of ideals of compact operators. Let $\mathcal{K}(Z, X)$ be an ideal in $\mathcal{W}(Z, X)$ for all reflexive Banach spaces $Z$. For a Banach space $Y$, let $G$ be a finite dimensional subspace of $\mathcal{W}(Y, X)$ and let $\varepsilon>0$. By Corollary 2.4, we can find a reflexive space 
$Z$, a norm one operator $J: Y \rightarrow Z$, and an isometry $\Phi$ taking $G$ into $\mathcal{W}(Z, X)$ and preserving compact operators such that $T=\Phi(T) \circ J$ for $T \in G$. By Theorem 3.2, there is an operator $A: \Phi(G) \rightarrow \mathcal{K}(Z, X)$ which "locally 1-complement" $\mathcal{K}(Z, X)$ in $\mathcal{W}(Z, X)$. Then $B: G \rightarrow \mathcal{K}(Y, X)$ defined by $B(T)=A(\Phi(T)) \circ J, T \in G$, "locally 1-complements" $\mathcal{K}(Y, X)$ in $\mathcal{W}(Y, X)$. This proves the claim about compact operators.

Now, if $\mathcal{F}(Z, X)$ is an ideal in $\mathcal{W}(Z, X)$ for all reflexive spaces $Z$, then, as we mentioned above, $X$ has the approximation property. Consequently, $\overline{\mathcal{F}(Y, X)}=\mathcal{K}(Y, X)$ for all Banach spaces $Y$ (cf. e.g. Remark 1.2). Therefore, by the first part of the proof, $\mathcal{F}(Y, X)$ is an ideal in $\mathcal{W}(Y, X)$ for all Banach spaces $Y$.

Remark 3.2. The assertion of Theorem 3.1 concerning ideals of finite rank operators can also be proved similarly to the case of ideals of compact operators in Theorem 3.1, using that the isometry from Corollary 2.4 preserves finite rank operators. However, in this case, one should apply Remark 3.1 and notice that the condition from Remark 3.1 works also for subspaces $F$ which are not necessarily closed.

In the next result, we summarize criteria of the approximation property expressed in termes of ideals of finite rank operators obtained in this paper and in the paper [25] by Lima and Oja.

Theorem 3.3. Let $X$ be a Banach space. The following statements are equivalent.

(i) $X$ has the approximation property.

(ii) $\mathcal{F}(Y, X)$ is an ideal in $\mathcal{W}(Y, X)$ for all Banach spaces $Y$.

(iii) $\mathcal{F}(Y, X)$ is an ideal in $\mathcal{W}(Y, X)$ for all separable reflexive Banach spaces $Y$.

(iv) $\mathcal{F}(Y, X)$ is an ideal in $\mathcal{W}(Y, X)$ for all closed subspaces $Y \subset c_{0}$.

(v) $\mathcal{F}(Y, X)$ is an ideal in $\mathcal{K}(Y, X)$ for all Banach spaces $Y$.

(vi) $\mathcal{F}(Y, X)$ is an ideal in $\mathcal{K}(Y, X)$ for all separable reflexive Banach spaces $Y$.

(vii) $\mathcal{F}(Y, X)$ is an ideal in $\mathcal{K}(Y, X)$ for all closed subspaces $Y \subset c_{0}$.

Proof. The equivalence (i) $\Leftrightarrow$ (ii) has just been proved above. The implications (vi) $\Rightarrow$ (i) and (vii) $\Rightarrow$ (i) are proved in [25, Theorem 5.1]. The other required implications (e.g. (ii) $\Rightarrow(\mathrm{v}) \Rightarrow$ (vi) \& (vii)) are obvious.

In the paper [25] by Lima and Oja, it was proved that interchanging the roles of $X$ and $Y$ in statements (v), (vi), and (vii) of Theorem 3.3 gives conditions equivalent to the approximation property of $X^{*}$. This 
result will be used and extended in the following symmetric version of Theorem 3.3.

Theorem 3.4. The following statements are equivalent:

(i) $X^{*}$ has the approximation property.

(ii) $\mathcal{F}(X, Y)$ is an ideal in $\mathcal{W}(X, Y)$ for all Banach spaces $Y$.

(iii) $\mathcal{F}(X, Y)$ is an ideal in $\mathcal{W}(X, Y)$ for all separable reflexive Banach spaces $Y$.

(iv) $\mathcal{F}(X, Y)$ is an ideal in $\mathcal{W}(X, Y)$ for all closed subspaces $Y \subset c_{0}$.

(v) $\mathcal{F}(X, Y)$ is an ideal in $\mathcal{K}(X, Y)$ for all Banach spaces $Y$.

(vi) $\mathcal{F}(X, Y)$ is an ideal in $\mathcal{K}(X, Y)$ for all separable reflexive Banach spaces $Y$.

(vii) $\mathcal{F}(X, Y)$ is an ideal in $\mathcal{K}(X, Y)$ for all closed subspaces $Y \subset c_{0}$.

Let us recall that, by a fundamental result due to Grothendieck [16] (cf. e.g. [26, p. 33]), $X^{*}$ has the approximation property if and only if $\overline{\mathcal{F}(X, Y)}=\mathcal{K}(X, Y)$ for all Banach spaces $Y$.

In the proof of Theorem 3.4, we shall need the following symmetric version of Theorem 3.1.

Theorem 3.5. Let $X$ be a Banach space. Then $\mathcal{F}(X, Y)($ resp. $\mathcal{K}(X, Y))$ is an ideal in $\mathcal{W}(X, Y)$ for all Banach spaces $Y$ if and only if $\mathcal{F}(X, Z)$ (resp. $\mathcal{K}(X, Z)$ ) is an ideal in $\mathcal{W}(X, Z)$ for all reflexive Banach spaces $Z$.

Proof. The case of compact operators can be proved as in Theorem 3.1 by applying Theorem 2.3 instead of Corollary 2.4.

Let $\mathcal{F}(X, Z)$ be an ideal in $\mathcal{W}(X, Z)$ for all reflexive spaces $Z$. Then, by the natural isometry $\left.T \rightarrow T^{*}\right|_{X}$ between $\mathcal{W}\left(Z^{*}, X^{*}\right)$ and $\mathcal{W}(X, Z)$, we have that $\mathcal{F}\left(Y, X^{*}\right)$ is an ideal in $\mathcal{W}\left(Y, X^{*}\right)$ for all reflexive Banach spaces $Y$, meaning that $X^{*}$ has the approximation property. Therefore, as we recalled above, $\overline{\mathcal{F}(X, Y)}=\mathcal{K}(X, Y)$ for all Banach spaces $Y$. And the already proved case of compact operators implies that $\mathcal{F}(X, Y)$ is an ideal in $\mathcal{W}(X, Y)$ for all Banach spaces $Y$.

Proof of Theorem 3.4. The equivalence (i) $\Leftrightarrow$ (ii) is clear from Theorem 3.5 and its proof. The implications (vi) $\Rightarrow$ (i) and (vii) $\Rightarrow$ (i) are proved in $[25$, Theorem 5.2], and the other required implications are obvious.

\section{The COMPACT APPROXimation PROPERTY AND IDEALS OF COMPACT OPERATORS}

Replacing the finite rank operators by compact operators gives the definition of the compact approximation property: one says that a Banach 
space $X$ has the compact approximation property (resp. the metric compact approximation property) if $I_{X}$ belongs to the closure of $\mathcal{K}(X, X)$ (resp. $\left.B_{\mathcal{K}(X, X)}\right)$ with respect to the topology of uniform convergence on compact subsets in $X$. It is known that even the metric compact approximation property does not imply the approximation property [32].

By the previous section, $X$ has the approximation property if and only if $\mathcal{F}(Y, X)$ is an ideal in $\mathcal{W}(Y, X)$ for all Banach spaces $Y$. We shall show that one can replace finite rank operators by compact operators in the "only if" part of this characterization (cf. Theorem 4.1), but one cannot do this in the "if" part (cf. the following example).

Example. There is a Banach space $X$ without the compact approximation property such that $\mathcal{K}(Y, X)=\mathcal{W}(Y, X)$ (i.e. $\mathcal{K}(Y, X)$ is trivially an ideal in $\mathcal{W}(Y, X))$ for all Banach spaces $Y$.

Let $X$ be a closed subspace of $\ell_{1}$ without the compact approximation property (cf. [31] or e.g. [27, p. 107]). If $T \in \mathcal{W}(Y, X)$ for a Banach space $Y$, then by the Eberlein-Smulian theorem and the Schur property of $\ell_{1}$, it follows that $T$ is compact.

Theorem 4.1. Let $X$ be a Banach space and let the number a be fixed so that $f(a)=1$. The following assertions are equivalent and they hold whenever $X$ has the compact approximation property.

(a) $\mathcal{K}(Y, X)$ is an ideal in $\mathcal{W}(Y, X)$ for all Banach spaces $Y$.

(b) $\mathcal{K}(Y, X)$ is an ideal in $\mathcal{W}(Y, X)$ for all separable reflexive Banach spaces $Y$.

(c) $\mathcal{K}\left(X_{K}, X\right)$ is an ideal in $\operatorname{span}\left(\mathcal{K}\left(X_{K}, X\right) \cup\left\{J_{K}\right\}\right)$ for every $K \in$ $\mathcal{W}_{X}$

(d) For every Banach space $Y$ and every $T \in \mathcal{W}(Y, X)$, there is a net $\left(T_{\alpha}\right)$ in $\mathcal{K}(Y, X)$ with $\sup _{\alpha}\left\|T_{\alpha}\right\| \leq\|T\|$ such that $T_{\alpha} y \underset{\alpha}{\longrightarrow}$ Ty for all $y \in Y$.

(e) For every separable reflexive Banach space $Y$ and every $T \in$ $\mathcal{W}(Y, X)$, there is a sequence $\left(T_{n}\right)$ in $\mathcal{K}(Y, X)$ with $\sup _{n}\left\|T_{n}\right\| \leq$ $\|T\|$ such that $T_{n} y \underset{n}{\rightarrow}$ Ty for all $y \in Y$.

(f) For every $K \in \mathcal{W}_{X}$, there is a net $\left(A_{\alpha}\right)$ in $B_{\mathcal{K}\left(X_{K}, X\right)}$ such that $A_{\alpha} x \underset{\alpha}{\longrightarrow} J_{K} x$ for all $x \in X_{K}$.

For the proof of Theorem 4.1, and also in the sequel, we shall need the following well-known description of duals of spaces of compact operators due to Feder and Saphar [12]. Let us recall that if $X$ and $Y$ are Banach spaces, then for any $v \in X^{*} \hat{\otimes}_{\pi} Y^{* *}, v=\sum_{n=1}^{\infty} x_{n}^{*} \otimes y_{n}^{* *}$ with 
$\sum_{n=1}^{\infty}\left\|x_{n}^{*}\right\|\left\|y_{n}^{* *}\right\|<\infty$, and for any $T \in \mathcal{L}(Y, X)$, the element $T^{* *} v \in$ $X^{*} \hat{\otimes}_{\pi} X^{* *}$ is defined by $T^{* *} v=\sum_{n=1}^{\infty} x_{n}^{*} \otimes T^{* *} y_{n}^{* *}$.

Lemma 4.2 (cf. [12, Theorem 1]). Let $X$ and $Y$ be Banach spaces such that $X^{*}$ or $Y^{* *}$ has the Radon-Nikodym property. Let $\Phi: X^{*} \hat{\otimes}_{\pi} Y^{* *} \rightarrow$ $\mathcal{L}(Y, X)^{*}$ be defined by

$$
(\Phi v)(T)=\operatorname{trace}\left(T^{* *} v\right), \quad T \in \mathcal{L}(Y, X), \quad v \in X^{*} \hat{\otimes}_{\pi} Y^{* *} .
$$

Then, for all $g \in \mathcal{K}(Y, X)^{*}$, there exists $v \in X^{*} \hat{\otimes}_{\pi} Y^{* *}$ such that $g=$ $\left.(\Phi v)\right|_{\mathcal{K}(Y, X)}$ and $\|g\|=\|\Phi v\|$.

Proof of Theorem 4.1. The implications (a) $\Rightarrow(\mathrm{b}),(\mathrm{a}) \Rightarrow(\mathrm{c})$, and (d) $\Rightarrow$ (f) are obvious. The implications (c) $\Rightarrow(\mathrm{f})$ and $(\mathrm{b}) \Rightarrow(\mathrm{e})$ are immediate from Lemma 1.3 (for $(\mathrm{b}) \Rightarrow$ (e), one should also use the standard argument from the proof of (iii) $\Rightarrow$ (iv) in Corollary 1.4). The proofs of (e) $\Rightarrow$ (f) and (f) $\Rightarrow$ (d) are essentially the same as, respectively, the proofs of (iv) $\Rightarrow$ (i) and (i) $\Rightarrow$ (iii) in Corollary 1.4.

(f) $\Rightarrow$ (a). We shall apply Theorem 3.2 together with Remark 3.1 to show that $\mathcal{K}(Y, X)$ is an ideal in $\mathcal{W}(Y, X)$. Let $G$ be a finite dimensional subspace of $\mathcal{W}(Y, X)$ and let $\varepsilon>0$. By Theorem 2.3, there exist $K \in \mathcal{W}_{X}$ and a linear isometry $\Phi: G \rightarrow \mathcal{W}\left(Y, X_{K}\right)$ preserving compact operators such that $T=J_{K} \circ \Phi(T)$ for all $T \in G$. Let a net $\left(A_{\alpha}\right)$ in $B_{\mathcal{K}\left(X_{K}, X\right)}$ satisfy $\left\|\left(A_{\alpha}-J_{K}\right) x\right\| \underset{\alpha}{\rightarrow} 0$ for all $x \in X_{K}$. Since $\left\{\Phi(T) y: T \in B_{G \cap \mathcal{K}(Y, X)}, y \in\right.$ $\left.B_{Y}\right\}$ is a relatively compact subset of $X_{K}$, there is an $\alpha$ so that $\|\left(A_{\alpha}-\right.$ $\left.J_{K}\right) \Phi(T) y \| \leq \varepsilon$ for all $T \in B_{G \cap \mathcal{K}(Y, X)}$ and $y \in B_{Y}$. This means that $\left\|A_{\alpha} \circ \Phi(T)-T\right\| \leq \varepsilon$ for all $T \in B_{G \cap \mathcal{K}(Y, X)}$. And denoting $A(T)=$ $A_{\alpha} \circ \Phi(T), T \in G$, we get an operator $A: G \rightarrow \mathcal{K}(Y, X)$ as desired.

Finally, let us assume that $X$ has the compact approximation property. We shall show that $\mathcal{K}(Y, X)$ is an ideal in $\mathcal{W}(Y, X)$ for any reflexive Banach space $Y$. To this end, we shall develop the proof of the implication (i) $\Rightarrow$ (ii) in Theorem 1.2 using instead of Grothendieck's representation theorem the description of $\mathcal{K}(Y, X)^{*}$ due to Feder and Saphar (see Lemma 4.2).

Consider $f \in \mathcal{L}(Y, X)^{*}$. For $g=\left.f\right|_{\mathcal{K}(Y, X)}$, let $v=\sum_{n=1}^{\infty} x_{n}^{*} \otimes y_{n} \in$ $X^{*} \hat{\otimes}_{\pi} Y$ with $\sum_{n=1}^{\infty}\left\|x_{n}^{*}\right\|<\infty$ and $\left\|y_{n}\right\| \rightarrow 0$ be given by Lemma 4.2. We assume that $\left(K_{\alpha}\right) \subset \mathcal{K}(X, X)$ converge to $I_{X}$ uniformly on the compact 
subsets of $X$. Then, for $T \in \mathcal{L}(Y, X)$,

$$
\begin{aligned}
\left|(\Phi v)(T)-f\left(K_{\alpha} T\right)\right| & =\left|(\Phi v)\left(T-K_{\alpha} T\right)\right| \\
& =\left|\sum_{n=1}^{\infty} x_{n}^{*}\left(\left(T-K_{\alpha} T\right) y_{n}\right)\right| \\
& \leq \sup _{n}\left\|\left(I_{X}-K_{\alpha}\right)\left(T y_{n}\right)\right\| \sum_{n=1}^{\infty}\left\|x_{n}^{*}\right\| \underset{\alpha}{\rightarrow} 0
\end{aligned}
$$

because $\left\{0, T y_{1}, T y_{2}, \ldots\right\}$ is a compact subset of $X$. Since $\Phi v \in \mathcal{L}(Y, X)^{*}$ is a norm-preserving extension of $\left.f\right|_{\mathcal{K}(Y, X)}$, the mapping $P: \mathcal{L}(Y, X)^{*} \rightarrow$ $\mathcal{L}(Y, X)^{*}$ defined by

$$
P(f)(T)=\lim _{\alpha} f\left(K_{\alpha} T\right)=(\Phi v)(T), \quad f \in \mathcal{L}(Y, X)^{*}, \quad T \in \mathcal{L}(Y, X),
$$

is a norm one projection with $\operatorname{ker} P=\mathcal{K}(Y, X)^{\perp}$.

Remark 4.1. Since $X_{K}$ is reflexive whenever $K \in \mathcal{W}_{X}$, Theorem $3.1 \mathrm{im}$ mediately follows from Lemma 1.3 and the implication (f) $\Rightarrow$ (a) of Theorem 4.1. However, the proof of Theorem 3.1 we gave in Section 3 is easier and more direct.

Remark 4.2. The idea to define a norm one projection with $\operatorname{ker} P=$ $\mathcal{K}(Y, X)^{\perp}$ on $\mathcal{L}(Y, X)^{*}$ by $(P f)(T)=\lim _{\alpha} f\left(K_{\alpha} T\right), f \in \mathcal{L}(Y, X)^{*}, T \in$ $\mathcal{L}(Y, X)$, whenever $K_{\alpha} \in B_{\mathcal{K}(Y, X)}$ and $K_{\alpha} \rightarrow I_{X}$, is due to J. Johnson [20]. In Theorem 4.1, the set of operators $K_{\alpha}$ is not necessarily bounded.

Remark 4.3. As we saw above, $\mathcal{F}(Y, X)$ is an ideal in $\mathcal{W}(Y, X)$ for all Banach spaces $Y$ whenever there exists a number $a>1$ so that $\mathcal{F}\left(X_{K}, X\right)$ is an ideal in $\mathcal{W}\left(X_{K}, X\right)$ for all $K \in \mathcal{W}_{X}$. By Theorem 4.1, (c) $\Rightarrow$ (a), the similar assertion for compact operators holds for the number $a$ for which $f(a)=1$.

We say that a Banach space $X$ has the weakly compact approximation property if $I_{X}$ belongs to the closure of $\mathcal{W}(X, X)$ with respect to the topology of uniform convergence on compact subsets in $X$. This notion was considered by Reinov [30] and by Grønbæk and Willis [17]. Note that Astala and Tylli [2] use this notion when $I_{X}$ belongs to the closure of $\mathcal{W}(X, X)$ with respect to the topology of uniform convergence on weakly compact subsets in $X$.

Corollary 4.3. The assertions of Theorem 4.1 are equivalent to the compact approximation property of $X$ whenever $X$ has the weakly compact approximation property. 
Proof. Let $K \in \mathcal{K}_{X}$, let $\varepsilon>0$, and choose $T \in \mathcal{W}(X, X)$ such that $\|T x-x\|<\varepsilon / 2$ for all $x \in K$. By assertion (d) of Theorem 4.1, there is a bounded net $\left(T_{\alpha}\right)$ in $\mathcal{K}(X, X)$ such that $T_{\alpha} x \rightarrow T x$ for all $x \in X$. By compactness of $K, \sup _{x \in K}\left\|T_{\alpha} x-T x\right\| \rightarrow 0$ and therefore $\sup _{x \in K} \| T_{\alpha} x-$ $x \|<\varepsilon$ for some $\alpha$.

Remark 4.4. Corollary 4.3 applies, in particular, to Banach spaces $X$ which are reflexive. However in this case, the assertions of Theorem 4.1 are equivalent to the metric compact approximation property of $X$ and also to the fact that $\mathcal{K}(X, X)$ is an ideal in $\mathcal{W}(X, X)$ (cf. [23, Theorem 14]).

Corollary 4.4. Let $X$ be a Banach space and let the number a be fixed so that $f(a)=1$. The following assertions are equivalent and they hold whenever $X^{*}$ has the compact approximation property.

(a) $\mathcal{K}(X, Y)$ is an ideal in $\mathcal{W}(X, Y)$ for all Banach spaces $Y$.

(b) $\mathcal{K}(X, Y)$ is an ideal in $\mathcal{W}(X, Y)$ for all separable reflexive Banach spaces $Y$.

(c) For every Banach space $Y$ and every $T \in \mathcal{W}(X, Y)$, there is a net $\left(T_{\alpha}\right)$ in $\mathcal{K}(X, Y)$ with $\sup _{\alpha}\left\|T_{\alpha}\right\| \leq\|T\|$ such that $T_{\alpha}^{*} y^{*} \underset{\alpha}{\longrightarrow} T^{*} y^{*}$ for all $y^{*} \in Y^{*}$.

(d) For every separable reflexive Banach space $Y$ and every $T \in$ $\mathcal{W}(X, Y)$, there is a sequence $\left(T_{n}\right)$ in $\mathcal{K}(X, Y)$ with $\sup _{n}\left\|T_{n}\right\| \leq$ $\|T\|$ such that $T_{n}^{*} y^{*} \underset{n}{\longrightarrow} T^{*} y^{*}$ for all $y^{*} \in Y^{*}$.

Proof. We shall use the natural isometry $\left.T \rightarrow T^{*}\right|_{X}$ between $\mathcal{W}\left(Z^{*}, X^{*}\right)$ and $\mathcal{W}(X, Z)$ for reflexive Banach spaces $Z$. By this isometry, $\mathcal{K}(X, Y)$ is an ideal in $\mathcal{W}(X, Y)$ for all reflexive Banach spaces $Y$ if and only if $\mathcal{K}\left(Y, X^{*}\right)$ is an ideal in $\mathcal{W}\left(Y, X^{*}\right)$ for all reflexive Banach spaces $Y$. Applying Theorems 3.5 and 3.1, this yields the equivalence of (a) to condition (a) of Theorem 4.1 for $X^{*}$. Furthermore, by the same isometry, (b) and (d) are respectively equivalent to conditions (b) and (e) of Theorem 4.1 for $X^{*}$, (c) implies condition (d) of Theorem 4.1 for $X^{*}$ which, in its turn, implies the particular case of (c) where $Y$ is assumed to be reflexive. Hence, by Theorem 4.1, (c) $\Rightarrow(\mathrm{a}) \Leftrightarrow(\mathrm{b}) \Leftrightarrow(\mathrm{d})$, the last equivalent conditions hold whenever $X^{*}$ has the compact approximation property, and they imply the particular case of (c) with reflexive $Y$. To finish the proof, we have to show that this particular case of (c) actually implies (c). Let $Y$ be a Banach space and let $T \in \mathcal{W}(X, Y)$. Let $K, Y_{K}, T_{K}$, and $J_{K}$ be as in Theorem 2.2. Since $K$ is weakly compact, $Y_{K}$ is reflexive. Hence, for $T_{K} \in \mathcal{W}\left(X, Y_{K}\right)$, there is a net $\left(S_{\alpha}\right)$ in $\mathcal{K}\left(X, Y_{K}\right)$ with $\sup _{\alpha}\left\|S_{\alpha}\right\| \leq\left\|T_{K}\right\|=\|T\|$ such that 
$S_{\alpha}^{*} z^{*} \underset{\alpha}{\rightarrow} T_{K}^{*} z^{*}$ for all $z^{*} \in Y_{K}^{*}$. Since $\left\|J_{K}\right\|=1$, the net $T_{\alpha}=J_{K} \circ S_{\alpha}$ clearly satisfies what is needed.

\section{From APPROXimation PROPERTIES TO METRIC APPROXIMATION PROPERTIES}

We would like to demonstrate how the method of proof of Theorem 1.2 can be further developed to give alternative proofs for known results about cases when the (compact) approximation property implies the metric (compact) approximation property. (Note that the following results could have been obtained already in Section 1, but by their nature, they fit more properly to conclude this paper.)

The dual space $X^{*}$ of a Banach space $X$ is said to have the compact approximation property with conjugate operators if $I_{X^{*}}$ belongs to the closure of $\left\{K^{*}: K \in \mathcal{K}(X, X)\right\}$ with respect to the topology of uniform convergence on compact subsets of $X^{*}$. By an example due to Grønbæk and Willis [17], the compact approximation property of $X^{*}$ does not imply the compact approximation property with conjugate operators. Moreover, Casazza and Jarchow [3] have shown that there is a Banach space $X$ failing the metric compact approximation property such that all its duals $X^{*}, X^{* *}, \ldots$ have the metric compact approximation property. Let us recall that if $X^{*}$ has the approximation property, then $X^{*}$ has the approximation property with conjugate operators (this is clear from the local reflexivity principle).

The following two results will explain surprisingly well why, in certain important cases, the (compact) approximation property implies the metric (compact) approximation property.

Theorem 5.1. Let $X$ and $Y$ be Banach spaces such that $X^{*}$ or $Y^{* *}$ has the Radon-Nikodym property. If $X^{*}$ has the compact approximation property with conjugate operators, then $\mathcal{K}(Y, X)$ is an ideal in $\mathcal{L}(Y, X)$ with an ideal projection $P$ such that

$$
P\left(y^{* *} \otimes x^{*}\right)=y^{* *} \otimes x^{*} \quad \text { for all } x^{*} \in X^{*} \text { and } y^{* *} \in Y^{* *} .
$$

Proof. We assume that $\left(K_{\alpha}^{*}\right)$ with $K_{\alpha} \in \mathcal{K}(X, X)$ converges to $I_{X^{*}}$ uniformly on compact subsets of $X^{*}$. Similarly to the proof of Theorem 4.1, we can define an ideal projection $P$ by

(*) $\quad(P f)(T)=\lim _{\alpha} f\left(T K_{\alpha}\right), \quad f \in \mathcal{L}(Y, X)^{*}, \quad T \in \mathcal{L}(Y, X)$.

In particular, for $f=y^{* *} \otimes x^{*}$ and $T \in \mathcal{L}(Y, X)$, this implies

$$
\left(P\left(y^{* *} \otimes x^{*}\right)\right)(T)=\lim _{\alpha} y^{* *}\left(K_{\alpha}^{*} T^{*} x^{*}\right)=y^{* *}\left(T^{*} x^{*}\right)=\left(y^{* *} \otimes x^{*}\right)(T) .
$$


Theorem 5.2. Let $X$ be a Banach space. The following statements are equivalent.

(a) $X^{*}$ has the metric compact approximation property with conjugate operators.

(b) For all Banach spaces $Y, \mathcal{K}(Y, X)$ is an ideal in $\mathcal{L}(Y, X)$ with an ideal projection $P$ such that

$P\left(y^{* *} \otimes x^{*}\right)=y^{* *} \otimes x^{*} \quad$ for all $x^{*} \in X^{*}$ and $y^{* *} \in Y^{* *}$.

(c) $\mathcal{K}(X, X)$ is an ideal in $\operatorname{span}(\mathcal{K}(X, X) \cup\{I\})$ with an ideal projection $P$ such that

$P\left(x^{* *} \otimes x^{*}\right)=x^{* *} \otimes x^{*} \quad$ for all $x^{*} \in X^{*}$ and $x^{* *} \in X^{* *}$.

Proof. (a) $\Rightarrow(\mathrm{b})$. Let $\left(K_{\alpha}\right)$ be a net in $B_{\mathcal{K}(X, X)}$ such that $K_{\alpha}^{*} x^{*} \rightarrow x^{*}$ for all $x^{*} \in X^{*}$. Applying a well-known result due to J. Johnson [20], by passing to a subnet of $\left(K_{\alpha}\right)$, one can define an ideal projection $P$ by $(*)$. As in the proof of Theorem 5.1, we have $P\left(y^{* *} \otimes x^{*}\right)=y^{* *} \otimes x^{*}$ for all $x^{*} \in X^{*}$ and $y^{* *} \in Y^{* *}$.

(b) $\Rightarrow$ (c). This is obvious.

$(\mathrm{c}) \Rightarrow(\mathrm{a})$. By Lemma 1.3, there exists a net $\left(K_{\alpha}\right)$ in $B_{\mathcal{K}(X, X)}$ such that

$$
x^{* *}\left(K_{\alpha}^{*} x^{*}\right) \underset{\alpha}{\longrightarrow} P\left(x^{* *} \otimes x^{*}\right)\left(I_{X}\right)=\left(x^{* *} \otimes x^{*}\right)\left(I_{X}\right)=x^{* *}\left(x^{*}\right)
$$

for all $x^{*} \in X^{*}$ and $x^{* *} \in X^{* *}$. Thus $K_{\alpha}^{*} \rightarrow I_{X^{*}}$ in the weak operator topology of $\mathcal{L}\left(X^{*}, X^{*}\right)$. Since the weak and strong operator topologies yield the same dual space, after passing to convex combinations, we may assume that $K_{\alpha}^{*} \rightarrow I_{X^{*}}$ in the strong operator topology.

As an immediate corollary of Theorems 5.1 and 5.2, we obtain the following result due to Godefroy and Saphar [15].

Corollary 5.3 (cf. [15, Corollary 1.6]). Let $X$ be a Banach space such that $X^{*}$ or $X^{* *}$ has the Radon-Nikodým property. If $X^{*}$ has the compact approximation property with conjugate operators, then $X^{*}$ has the metric compact approximation property with conjugate operators.

Remark 5.1. The original proof of Corollary 5.3 due to Godefroy and Saphar [15] was also based, like ours, on Lemma 4.2, but by using the local reflexivity principle, it was modeled after Grothendieck's classical proof in [16]. Another proof of Corollary 5.3 (under the assumption that $X^{*}$ has the Radon-Nikodým property) is given by Cho and Johnson [4] by an adaption of the alternative proof due to Lindenstrauss and Tzafriri [26, pp. 39-40]. 
The similar argument as in Theorem 5.2 yields the next result.

Theorem 5.4. Let $X$ be a Banach space. The following statements are equivalent.

(a) $X$ has the metric compact approximation property.

(b) For all Banach spaces $Y, \mathcal{K}(Y, X)$ is an ideal in $\mathcal{L}(Y, X)$ with an ideal projection $P$ such that

$$
P\left(y \otimes x^{*}\right)=y \otimes x^{*} \quad \text { for all } x^{*} \in X^{*} \text { and } y \in Y .
$$

(c) $\mathcal{K}(X, X)$ is an ideal in $\operatorname{span}(\mathcal{K}(X, X) \cup\{I\})$ with ideal projection $P$ such that

$$
P\left(x \otimes x^{*}\right)=x \otimes x^{*} \quad \text { for all } x^{*} \in X^{*} \text { and } x \in X .
$$

The equivalence $(\mathrm{a}) \Leftrightarrow(\mathrm{c})$ of Theorem 5.4 is contained in $[10$, Proposition 4].

An immediate corollary of Theorem 5.4 and Lemma 1.3 is the following result due to Lima [23].

Corollary 5.5 (cf. [23, Theorem 14]). Let $X$ be a Banach space with the Radon-Nikodym property. $X$ has the metric compact approximation property if and only if $\mathcal{K}(X, X)$ is an ideal in $\operatorname{span}(\mathcal{K}(X, X) \cup\{I\})$.

Theorems 5.2 and 5.4 remain valid for the metric approximation property if one replaces $\mathcal{K}(Y, X)$ by $\mathcal{F}(Y, X)$ and $\mathcal{K}(X, X)$ by $\mathcal{F}(X, X)$ (this is clear from the proofs). Therefore we have the following modifications of Corollaries 5.3 and 5.5.

Corollary 5.6 (cf. [8, p. 246]). Let $X$ be a Banach space such that $X^{*}$ or $X^{* *}$ has the Radon-Nikodym property. If $X^{*}$ has the approximation property, then $X^{*}$ has the metric approximation property.

Corollary 5.7 (cf. [23, Theorem 13]). Let $X$ be a Banach space with the Radon-Nikodym property. $X$ has the metric approximation property if and only if $\mathcal{F}(X, X)$ is an ideal in $\operatorname{span}(\mathcal{F}(X, X) \cup\{I\})$.

There are several important results on the (metric) approximation property for which it is not known whether or not they hold in the case of the (metric) compact approximation property. For instance, it is known, as we already mentioned above, that the (metric) approximation property for $X^{*}$ implies the same for $X$. Casazza and Jarchow [3] have shown that this is not true for the metric compact approximation property, but it seems to be an open question whether or not this is true for the compact approximation property. It is not known whether Corollary 5.3 remains 
true if $X^{*}$ has the compact approximation property (and not necessarily the compact approximation property with conjugate operators) (this question was posed by Godefroy and Saphar in [15]). It is known that the metric approximation property is separably determined: $X$ has the metric approximation property whenever every separable subspace is contained in a separable subspace of $X$ with the metric approximation property. In [28] (see also [29]), similar results were shown for the metric approximation property having some special geometric features (like unconditionality). We do not know whether these results hold for the metric compact approximation property.

\section{REFERENCES}

[1] D. Amir And J. Lindenstrauss. The structure of weakly compact sets in Banach spaces. Ann. Math. 88 (1968) 35-46.

[2] K. Astala And H.O. Tylli. Seminorms related to weak compactness and to Tauberian operators. Math. Proc. Camb. Phil. Soc. 107 (1990) 367-375.

[3] P.G. Casazza and H. Jarchow. Self-induced compactness in Banach spaces. Proc. R. Soc. Edinburgh Sect. A 126 (1996) 355-362.

[4] C.M. Cho And W.B. Johnson. A characterization of subspaces $X$ of $\ell_{p}$ for which $K(X)$ is an $M$-ideal in $L(X)$. Proc. Amer. Math. Soc. 93 (1985) 466-470.

[5] W.J. Davis, T. Figiel, W.B. Johnson, and A. Pęczyński. Factoring weakly compact operators. J. Funct. Analysis 17 (1974) 311-327.

[6] J. Diestel. Geometry of Banach Spaces - Selected Topics. Lecture Notes in Mathematics 485 Springer-Verlag, Berlin-Heidelberg-New York (1975).

[7] J. Diestel. Sequences and Series in Banach Spaces. Graduate Texts in Mathematics 92 Springer-Verlag, (1984).

[8] J. Diestel and J.J. Uhl, JR. Vector Measures. Mathematical Surveys 15, American Mathematical Society, Providence, (1977).

[9] N. Dunford And J.T. Schwartz. Linear Operators. Part 1: General Theory. Wiley Interscience (1958).

[10] G. Emmanuele And K. John. Some remarks on the position of the space $\mathcal{K}(X, Y)$ inside the space $\mathcal{W}(X, Y)$. New Zealand J. Math. 26 (1997) 183-189.

[11] H. Fakhoury. Sélections linéaires associées au théorème de Hahn-Banach. J. Funct. Analysis 11 (1972) 436-452.

[12] M. Feder And P.D. SAPhar. Spaces of compact operators and their dual spaces. Israel J. Math. 21 (1975) 38-49.

[13] T. Figiel. Factorization of compact operators and applications to the approximation problem. Studia Math. 45 (1973) 191-210.

[14] G. Godefroy, N.J. Kalton, and P.D. Saphar. Unconditional ideals in Banach spaces. Studia Math. 104 (1993) 13-59.

[15] G. Godefroy And P.D. Saphar. Duality in spaces of operators and smooth norms on Banach spaces. Illinois J. of Math. 32 (1988) 672-695.

[16] A. Grothendieck. Produits tensoriels topologiques et espaces nucléaires. Mem. Amer. Math. Soc. 16 (1955). 
[17] GrønbæK And Willis. Approximate identities in Banach algebras of compact operators. Canad. Math. Bull. 36 (1993) 45-53.

[18] P. Harmand, D. Werner, and W. Werner. M-ideals in Banach Spaces and Banach Algebras. Lecture Notes in Math. 1547, Springer-Verlag (1993).

[19] H. Jarchow. Locally Convex Spaces. B.G. Teubner Stuttgart (1981).

[20] J. Johnson. Remarks on Banach spaces of compact operators. J. Funct. Analysis. 32 (1979) 304-311.

[21] W.B. Johnson. Factoring compact operators. Israel J. Math. 9 (1971) 337-345.

[22] N.J. KALton. Locally complemented subspaces and $L_{p}$-spaces for $0<p<1$. Math. Nach. 115 (1984) 71-97.

[23] A. LimA. The metric approximation property, norm-one projections and intersection properties of balls. Israel J. Math. 84 (1993) 451-475.

[24] A. Lima. Property $\left(w M^{*}\right)$ and the unconditional metric compact approximation property. Studia Math. 113 (1995) 249-263.

[25] A. Lima AND E. OJA. Ideals of finite rank operators, intersection properties of balls, and the approximation property. Studia Math. 133 (2) (1999) 175-186.

[26] J. Lindenstrauss and L. Tzafriri. Classical Banach Spaces I. Ergebnisse der Mathematik und ihrer Grenzgebiete 92, Springer-Verlag (1977).

[27] J. Lindenstrauss And L. Tzafriri. Classical Banach Spaces II. Ergebnisse der Mathematik und ihrer Grenzgebiete 97, Springer-Verlag (1979).

[28] E. OJA. Geometry of Banach spaces having shrinking approximations of the identity. Trans. Amer. Math. Soc. (to appear)

[29] E. OJA. Géométrie des espaces de Banach ayant des approximations de l'identité contractantes C. R. Acad. Sci. Paris, Sér. I, 328 (1999) 1167-1170.

[30] O.I. REINOV How bad can a Banach space with the approximation property be? Mat. Zametki 33 (1983) 833-846 (in Russian); English translation in Math. Notes 33 (1983) 427-434.

[31] A. Szankowski. Subspaces without approximation property. Israel J. Math. 30 (1978) 123-129.

[32] G. WiLlis. The compact approximation property does not imply the approximation property. Studia Math. 103 (1992) 99-108.

[33] P. WojtaszczyK. Banach Spaces for Analysts. Cambridge Studies in Advanced Mathematics 25, Cambridge University Press (1991).

Deparment of Mathematics, Agder College, Tordenskjoldsgate 65, 4604 Kristiansand, Norway, Deparment of Mathematics, Agder College, TordenSkJoldsgate 65, 4604 Kristiansand, Norway, Faculty of Mathematics, Tartu University, VANEMUise 46, EE-2400 TARTu, Estonia

E-mail address: Asvald.Lima@hia.no, Olav.Nygaard@hia.no, eveoja@math.ut.ee 